2. Carson, S., Goldhamer, R., and Weinberg, M. S.: In: S. Jakowska: Interdisciplinary investigation of mucus product and transport. Ann. N.Y. Acad. Sci., 130(3) (1966)

3. Cheung, A. T. W.: Doctoral Thesis, 1973. University of California at Los Angeles.

4. Cheung, A. T. W., and Jahn, T. L.: Determination of the movement pattern of the epithelial cilia of rabbit trachea and the clearance mechanism of the tracheal muco-ciliary system. In: Proceedings of Symposium on Swimming and Flying in Nature, 1974 (California Institute of Technology. Pasadena. Calif., in press).

5. Cheung, A. T. W., and Wu, T. Y.: High speed cinemicrographic studies on rabbi tracheal (ciliated) epithelia: Biomechanics of tracheal mucus clearance (In preparation).

6. Falk, H. L., Kotin, P., and Rowlette, W.: In: Jakowska: Mucous secretion. Ann. N.Y. Acad. Sci. $106(2)(1963)$.

7. Fawcett, D. W., and Porter, K. R.: A study of the fine structure of ciliated epithelia. J. Morphol., 94:21 (1954)

8. Finerty, J. C., and Cowdry, E. V.: A Textbook of Histology, Ed. 5 (Lea \& Febiger, Philadelphia, 1960)

9. Gray, J.: Ciliary Movement (Cambridge University Press, Cambridge, England, 1928).

Copyright $(1976$ International Pediatric Research Foundation, Inc.
10. Greep, R. O. (Editor): Histology, Ed. 2 (McGraw-Hill Book Co., New York, 1966).

11. Lucas, A. M., and Douglas, L. C.: Principles underlying ciliary activity in the respiratory tract. Arch. Otolaryngol., 20: 518 (1934).

12. Zeiss Publications: Phase Contrast and Interference Contrast (Carl Zeiss Publications, West Germany).

13. We would like to express our deep gratitude to Professor Theodore $\mathrm{Y}$. $\mathrm{Wu}$, Professor Stuart R. Keller, and Dr. Christopher Brennen for their extremely valuable discussions in biophysical fluid mechanics. This work was jointly sponsored by the Office of Naval Research, National Science Foundation, and the United States Public Health Service. Their continous support is gratefully acknowledged.

14. This work is sponsored primarily by National Science Foundation Grant No. GK $31161 \mathrm{X}$ and Office of Naval Research Contract No. N00014-67-A-00940012 at the California Institute of Technology, and in part by National Science Foundation Grant No. GB 24840 and United States Public Health Service Grant No. AM 14388 at the University of California at Los Angeles.

15. Requests for reprints should be addressed to: A. T. W. Cheung, Ph.D. Thomas Laboratory (Mail Code: 104-44), California Institute of Technology, Pasadena, Calif. 91125 (USA).

16. Accepted for publication October 14, 1975

Printed in U.S.A

Pediat. Res. 10: 144-147 (1976)

Cinemicrography cystic fibrosis epithelial

tracheal cilia

\title{
High Speed Cinemicrographic Studies on Rabbit Tracheal (Ciliated) Epithelia: Cytolytic Effect of Cystic Fibrosis Serum on Tracheal Epithelial Cells
}

\author{
ANTHONY T. W. CHEUNG \\ California Institute of Technology, Pasadena, California, USA \\ THEODORE L. JAHN \\ University of California, Los Angeles, California, USA
}

\section{Extract}

High speed cinemicrographs are made on the ciliary activity of rabbit tracheal cilia with the aid of Nomarski optics. The detailed nature of the ciliary beat pattern is determined from slow motion analysis of the high speed cinemicrographs $(1-3)$. Such documented forms of ciliary beat pattern and the physiologic state of the tracheal epithelia are utilized as basic reference controls to investigate the physiologic effects of cystic fibrosis serum on ciliated epithelia and the cystic fibrotic tracheal mucus stagnation phenomenon.

Careful analyses of the high speed cinemicrographs reveal that cystic fibrosis serum has no effect on the rhythm and beat pattern of rabbit tracheal cilia. However, it is shown in controlled procedures that cystic fibrosis serum has a cytolytic effect on the tracheal epithelial cells and also at the cell junctions. Ciliary dyskinesis, as described in previous reports $\left(6_{-9}\right)$, is actually a secondary effect of cytolysis and cell destruction.

\section{Speculation}

Tracheal mucus stagnation in cystic fibrosis is not a ciliary dyskinetic problem, but rather a hydrodynamic and biochemical one.

Cystic fibrosis is a pediatric disorder which exhibits a very broad and varied spectrum of symptoms. One of the most notable is the stagnation of tracheal mucus transport. Some research reports had described the apparent presence of a cystic fibrosis factor in the serum of cystic fibrotic patients; such a factor was assumed to be the cause of ciliary dyskinesis and asynchrony in the beat pattern of tracheal cilia which could subsequently cause a stagnation of tracheal mucus transport (6-9). However, such a factor had not been satisfactorily defined and the apparent dyskinetic effect on ciliary activity had never been repeated outside of these laboratories. What made the situation more complicated was that the actual detailed beat pattern of tracheal cilia was still unknown and all the above reports were based solely on visual observations without any reference control.

This report follows up on the high speed cinemicrographic technique which has been utilized in the documentation and determination of the beat pattern of rabbit tracheal cilia $(1-3)$ and describes a method which serves to investigate the possible physiologic effects of cystic fibrosis serum on tracheal epithelial cells.

\section{MATERIALS AND METHODS}

\section{BIOLOGICAL MATERIAL}

Rabbit tracheal epithelia were obtained from New Zealand White rabbits purchased from biologic supply firms in Southern California. The rabbits were anesthetized by int ravenous injections of sodium pentobarbital (Diabutal) in a dosage dependent on body 
weights. The ciliated tracheal epithelia were microdissected out from the trachea and were suspended immediately in culture medium 199 (enriched with glutamine and Hank's salt). Under ideal conditions, the tracheal epithelial culture could be kept alive for over a week.

\section{SEROLOGIC MATERIAL}

Blood samples were obtained from cystic fibrosis patients as well as their parents at the UCLA-Cystic Fibrosis Clinic. Cystic fibrosis (homozygous) serum and cystic fibrotic carrier (heterozygous) serum were obtained from the available blood samples by standard clinical methods. Control serum was obtained from blood samples of established noncarriers of the disease.

\section{CHEMICAL MATERIAL}

Culture medium 199 was obtained from Grand Island Biological Company in Berkeley, California. The medium was stored at $4^{\circ}$ and buffered at $\mathrm{pH} 7.4$. The culture medium was enriched with glutamine and Hank's salt.

\section{OPTICAL EQUIPMENT}

Camera. Two 16-mm high speed cameras were used: a Redlake Locam high speed camera, model 162-4DC, and a Milliken Teledyne high speed camera, mode DBM-55. Both cameras were of pin registry and could manage an operating speed of 500 frames/sec.

Microscope. The movie camera was attached to a Zeiss research microscope (either a Zeiss Universal or a Zeiss Standard) equipped with Nomarski differential intereference contrast, phase contrast, and dark field optics. This high speed cinemicrographic set-up has been described in detail in previous reports $(1-4)$.

Light Source. Two different light sources were used: A tungstenhalogen 100-W source (at UCLA) and a Chadwick-Helmuth camera-modulated xenon strobe (at Caltech).

Timing Light. The exact framing rate of the high speed cinemicrographs could be determined by an internal timing light which was projected onto the edge of the film as a reference record.

Film. Kodak 4X negative $72244 \mathrm{XN}$ films and Kodak RAR stock 2475 recording films were normally used. Framing rates of up to $500 \mathrm{frames} / \mathrm{sec}$ could be used for these types of fast film.

Movie Analyzer. Analyses of the high speed cinemicrographs were made by repeated viewing at various speeds (2-24 frames/ sec) with a flicker-less L. W. photo-optical analyzer and also by utilizing a frame-by-frame tracing analytic procedure with the aid of a Traid R-100 film reader. Black and white photographic prints were made from selected movie sequences for analytic purposes.

\section{METHODS}

Microdissected tracheal epithelia (ciliated) were suspended in enriched culture medium 199. Segments with active ciliary movement and smooth cell margins were selected and placed under a Vaseline mount to be photographed with the high speed cinemicrographic set-up under Nomarski optics. The films were developed and processed in our own laboratory. Acufine was used in the developing process to yield a high ASA of over 3,000. Subsequent analyses of the high speed cinemicrographs served to illustrate the detailed form of tracheal ciliary beat pattern (1-3). This documented form of tracheal ciliary beat pattern was utilized as a reference control to investigate the phenomenon of ciliary asynchrony and dyskinesis.

For the investigation of the possible physiologic effects of the various sera on tracheal epithelia, a four-chambered slide was designed and utilized. A healthy piece of ciliated tracheal epithelium in $10 \mu \mathrm{l}$ culture medium was introduced into each of the four chambers. Ten microliters of culture medium were added into one chamber as a normal medium control, while $10 \mu \mathrm{l}$ normal serum were added into a second chamber as a serum control. The effects of the cystic fibrosis serum and that of the cystic fibrotic carrier (heterozygous) serum were then observed and documented by cinemicrography after the introduction of $10 \mu \mathrm{l}$ of each of the two respective sera separately into the two remaining chambers of the slide. Comparison with the controls could be carried out simultaneously. This four-chambered slide set-up minimized all possible experimental variations, and enabled the investigators to conduct the research on the same time schedule. Experimental results were disregarded totally if the ciliary activity of any one of the two controls showed any signs of irregularity or incoordination, or if the controls stopped beating in less than $4 \mathrm{hr}$. The fact that such ciliated epithelia were extremely sensitive and could sometimes beat asynchronously under some experimental conditions even without the introduction of sera made the utilization of such strict controls absolutely essential.

All interpretations and conclusions were based on analysis of the available cinemicrographs, thus eliminating the existence of any debatable human error in observation.

\section{RESULTS AND OBSERVATIONS}

The form of beat of tracheal cilia is determined from slow motion analysis of the high speed cinemicrographs taken with Nomarski optics (1-3). This documented form of ciliary beat pattern is utilized as a reference control for later investigations on the physiologic effects of cystic fibrosis serum on ciliated epithelial cells.

Twenty-five cystic fibrosis serum samples (from 25 different patients) and 9 cystic fibrotic carrier (heterozygous) serum samples were divided into 45 aliquots for 45 separate runs. The results on 6 of the trials were disregarded as the controls were not consistent in behavior. In the 39 controlled cases, the ciliary beat pattern and ciliary coordination (synchrony) were not hindered in any way after the introduction of the serum samples. Visual observations under Nomarski optics as well as subsequent analysis of the high speed cinemicrographs taken at various time intervals during the experimentation confirmed the fact that during 37 of the 39 cases, cystic fibrosis serum as well as cystic fibrotic carrier (heterozygous) serum had no dyskinetic effect on the beat pattern and coordination of tracheal cilia.

However, analysis of the cinemicrographs (in 35 of the 39 cases) also revealed that the tracheal cilia which had been treated with cystic fibrosis serum stopped beating about $2 \frac{1}{4} \mathrm{hr}$ after the introduction of the serum (as opposed to the absence of such a ciliary cessation in the two controls and the cystic fibrotic carrier sera trials). In about $1 \mathrm{hr}$, cellular debris started to appear at the cell junctions of the tracheal epithelia. The frequency of beat of the tracheal cilia, which was about $20-25 \mathrm{~Hz}$ under normal conditions, also started to slow down progressively. Apparently, some cytolytic factors had acted directly on the epithelial cells or at the cell junctions causing the loss of the regular shape of the epithelial cells, the appearance of cellular surface undulations, and the accumulation of cellular debris at the cell junctions (as shown in Fig. 1). The cytolysis at the cell junctions became more prominent in the course of time and in about $2 \mathrm{hr}$ detached and deformed epithelial cells could occasionally be seen floating around neighboring cells in the medium with the cilia still beating in good coordination. The ultimale cessation of the ciliary beat after $2^{1 / 4} \mathrm{hr}$ could be attributed as a secondary effect of cytolysis and cell destruction. The ciliary activity and the healthy physiologic state of the epithelia of both controls and that of the cystic fibrotic carrier (heterozygous) serum remained normal throughout.

\section{DISCUSSION}

Repeated analyses of the high speed cinemicrographs confirm the fact that cystic fibrosis serum and cystic fibrotic carrier (heterozygous) serum have no visible effect on the ciliary beat pattern and coordination of the tracheal cilia. The ciliary dyskinetic effect, as described by some investigators (6-9), is not visible in most of the serum runs. The discrepancy is probably due to the reliability of the controls used, visual and optical problems $(1,3)$, 


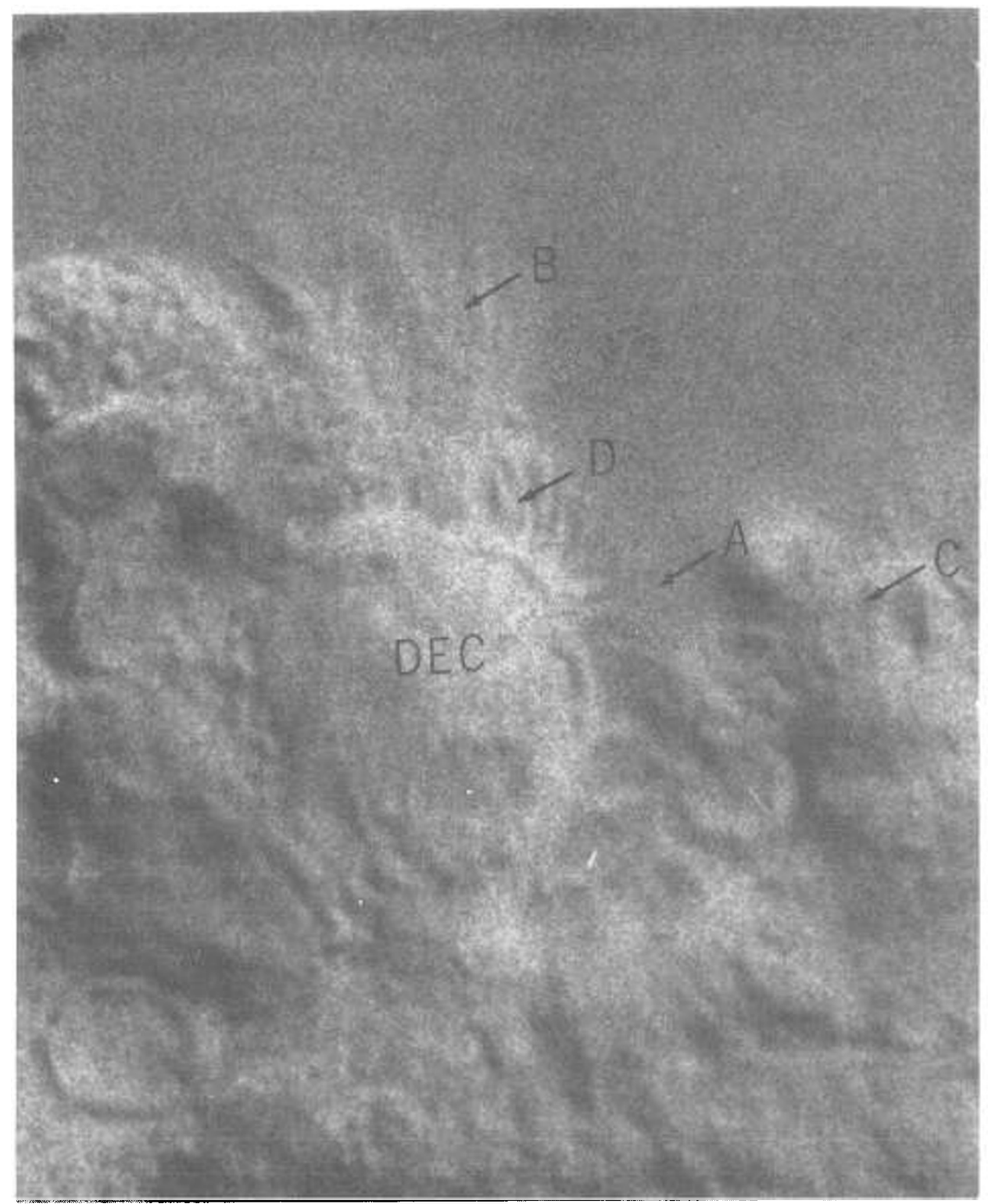

Fig. 1. A print of a segment of rabbit tracheal (ciliated) epithelium (about $2 \mathrm{hr}$ after the introduction of cystic fibrosis serum) from a selected 16-mm high speed movie sequence. $D E C$ denotes the detached epithelial cell floating in the medium. Marker $A$ indicates the original position of the epithelial cell before cytolysis; markers $B$ and $C$ point to the cilia of the original neighboring epithelial cells. Marker $D$ points to the cilia of the detached epithelial cell. The movie sequence shows that all the cilia are still beating actively and in good coordination. The blurriness of the print is due to the accumulation of cellular debris and the distortion of the cell surface which make sharp focusing of the movie sequence (under Nomarski optics) virtually impossible. Note that the margin and the surface of the detached epithelial cell have become quite distorted and irregular.

and the uncertainty in the standard detail beat pattern of tracheal cilia. The establishment of a good control system in the utilization of a four-chambered slide to eliminate experimental variations and the incorporation of a high speed cinemicrographic set-up with Nomarski optics can serve to minimize most problems.

Cystic fibrosis serum has a cytolytic effect on the tracheal epithelial cells; this fact is strongly supported by the accumulation of cellular debris at the cell junctions, the distortion of the regular shape of the epithelial cells, the appearance of cellular surface undulations, and the detachment of distorted epithelial cells after the introduction of cytic fibrosis serum.

Deactivation of the cytolytic property of the cystic fibrosis serum after heating at $56^{\circ}$ for $30 \mathrm{~min}$ raises the speculation that this cytolytic effect may be complement related, either through an abnormality in the control mechanism or the complements themselves. However, more research must be conducted along this line to look deeper into this cytolytic phenomenon before reaching any definite conclusions.
Since it is documented and proven that ciliary dyskinesis does not take place and consequently is not responsible for tracheal mucus stagnation in cystic fibrosis, an understanding of the ciliary beat pattern, the biochemistry of the mucous layer of the tracheal mucociliary system, and the biomechanics of its transport may serve to reveal the possible causes of this mucus stagnation phenomenon.

Analysis of previously obtained cinemicrographs $(1-3,5)$ reveals that the cilia of tracheal cilia are very short and stout (about $6 \mu \mathrm{m}$ in length) and that they beat in a planar forward-and-return manner that differs from classic concepts.

The original postural configuration of the cilia at the start of their forward strokes is slightly bent and the forward stroke starts at a near vertical position bearing about $75-90^{\circ}$ to the epithelial surface in the direction of the forward stroke. The forward stroke consists of a simple "bowing" movement, sweeping through an arc of about $40^{\circ}$ (with the tips of the cilia penetrating the bottom of the mucous layer for the first $5-8^{\circ}$ of the forward sweep), and in 
so doing serves to "scoop" up a column of liquid with each forward stroke. The rigid and stout nature of the cilia, the unconventional "bowing" forward stroke, the extent of the angular sweep, the near vertical starting position, and the original bent ciliary configuration all serve to enhance the efficiency in moving the surrounding liquid forward. The return stroke, which is slower and returning from close to the epithelial surface to a near vertical position, serves to minimize the dragging back of the surrounding liquid and the mucous layer on top of it $(1-3,5)$.

With each forward stroke the swift movement of the intermediate serous liquid layer interacts hydrodynamically with the viscoelastic mucous layer on top of it. This fricitional intermolecular interaction serves to cause the top mucous layer to move along with the intermediate serous liquid layer in the direction of the forward stroke. The "clawing" action of the cilia tip penetration at the botton of the mucous layer at the start of each forward stroke also helps in moving the mucous layer forward. The relatively small dragging back of the intermediate serous liquid layer, as confirmed by fluid tracers $(1-3,5)$, does not cause any noticeable dragging back of the mucous layer.

The movement of the mucous layer is very similar in nature to the movement of a "conveyor belt" system $(1,2,5)$. The main driving force to cause the mucus transport is powered by each forward stroke of the tracheal cilia, with the intermediate liquid layer taking over the part of the rubber rollers and with the mucous layer moving in the manner of a conveyor belt. Any foreign particles or liquid droplets that are on top of the mucous layer will be transported and escaluated out of the trachea.

Mucus is a glycoprotein and it chemically behaves as an ion exchanger, becoming extremely viscous and nonelastic in abnormal ionic conditions. Such an abnormal condition can be found in cystic fibrotic cases and consequently the mucous layer of the tracheal mucociliary system becomes extremely viscous and nonelastic. The intermolecular frictional interaction between the mucous layer and the intermediate liquid layer is hindered and the mucus transport simply becomes stagnant, although the tracheal cilia are still functioning.

\section{SUMMARY}

Cystic fibrosis serum does not cause ciliary dyskinesis and incoordination as previously postulated. The tracheal mucus stagnation in cystic fibrosis is a biochemical and hydrodynamic problem, and is not cilia related. Further research is now being conducted in the Biophysical Fluidmechanics Laboratory at the California Institute of Technology to look deeper into this mucus clearance and stagnation problem.

\section{REFERENCES AND NOTES}

1. Cheung, A. T. W.: Doctoral thesis, 1973. University of California at Los Angeles.

2. Cheung, A. T. W., and Jahn, T. L.: Determination of the movement pattern of the epithelial cilia of rabbit trachea and the clearance mechanism of the tracheal muco-ciliary clearance system. Proceedings of Symposium on Swimming and Flying in Nature, 1974 (California Institute of Technology, Pasadena, in press).

3. Cheung, A. T. W., and Jahn, T. L.: High speed cinemicrographic studies on rabbit tracheal (ciliated) epithelia. I. Determination of the beat pattern of trachealcilia (In press)

4. Cheung, A. T. W., and Winet, H. (1974) Flow velocity profile over a ciliated surface. Proceedings of Symposium on Swimming and Flying in Nature, 1974 (California Institute of Technology, Pasedena, in press).

5. Cheung, A. T. W., and Wu, T. Y.: High speed cinemicrographic studies on rabbit tracheal (ciliated) epithelia: Biomechanics of tracheal mucus clearance (In preparation).

6. Conover, J., Bonforte, R. J., Hathaway, P., Paciuc, S., Conod, E. J., Hirschhorn, K., and Kopel, F. B.: Studies on ciliary dyskinesia factor in cystic fibrosis. I. Bioassay and heterozygote detection in serum. Pediat. Res. 7: 220 (1973).

7. Kopel, F. B., and Conover, J.: An evaluation of the rabbit tracheal cilia assay for cystic fibrosis. Ann Cystic Fibrosis Club Abstr. p. 32 (1972)

8. Spock, A., Heick, H. M. C., Cress, H., and Logan, W. S.: Abnormal serum factor in patients with cystic fibrosis of the pancreas. Pediat. Res., I: 173 (1967).

9. Spock, A., Heick, H. M. C., Cress, H., and Logan, W. S.: In vitro study of ciliary motility to detect individuals with active cystic fibrosis and carriers of the disease. Mod. Probl. Pediat., 10: 200 (1967)

10. We want to take this opportunity to express our appreciation for the generous assistance of Dr. Alan Osher and other coworkers in the Cystic Fibrosis Clinic of the UCLA Medical Center. It was with their cooperation and assistance that made this research project a reality. This work was jointly sponsored by the Department of the Navy (Office of Naval Research), National Science Foundation, and the United States Public Health Service. Their continuous support is gratefully acknowledged.

11. This work was sponsored primarily by National Science Foundation Grant no. GK $31161 X$ and Office of Naval Research Contract no. N00014-67-A-0094-0012 at the California Institute of Technology and in part by National Science Foundation Grant no. GB 24840 and United States Public Health Service Grant no. AM 14388 at the University of California at Los Angeles.

12. Requests for reprints should be addressed to: A. T. W. Cheung, Ph.D., Thomas Laboratory (mail code: 104-44), California Institute of Technology, Pasadena, Calif. 91125 (USA)

13. Accepted for publication October 14, 1975. 